\title{
The cosmological information on neutrino mixing
}

\author{
Pasquale Di Bari* \\ Deutsches Elektronen-Synchrotron DESY, 22603 Hamburg, Germany \\ E-mail: 'dibari@mail. desy.dé
}

ABstract: Cosmology provides interesting information on neutrino mixing models with sterile neutrinos. In this case non standard BBN effects can be relevant. We show how the recent measurement of the baryon content from the observations of CMB anisotropies together with the primordial nuclear abundances measurements can be used to constrain them. In particular four neutrino mixing models are potentially at variance with the cosmological observations. We also discuss the possible scenarios from future experiments.

\section{Constraints on non standard BBN models}

Evidences for neutrino mixing have been found both in atmospheric neutrino and in solar neutrino data and together they provide the first solid indication of new physics in Earth experiments. Hints of new physics were already present also in cosmology. For example the measurement of a total matter content larger than the baryonic one pointing to the existence of a new type of matter. The baryonic content was inferred from the model of standard Big Bang Nucleosynthesis (SBBN) using the primordial nuclear abundances measurements. The recent high resolution observations of CMB anisotropies have confirmed the existence of acoustic peaks in the power spectrum as predicted by earlier theoretical studies that also relate their features to the values of the cosmological parameters. In this way it has already been possible to get a measurement of the baryon content independently on SBBN. This measurement relies on some minimal assumptions, but it is quite stable respect to the relaxation of many of them. The agreement, within the errors, with the value inferred from SBBN, strongly supports the existence of non baryonic dark matter and can be used to constrain a large variety of non standard BBN effects. We are interested in those non standard BBN effects that arise in neutrino mixing models and we want to see which kind of information can be extracted from current cosmological observations on them ${ }^{1}$. From the observation of CMB anisotropies, the BOOMERanG and DASI experiments find for the baryon contribution to the energy density $\left(\Omega_{b} h^{2}\right)^{C M B}=0.022_{-0.003}^{+0.004}$,

${ }^{*}$ Speaker.

${ }^{1}$ For details see [1] 
that implies a value of baryon to photon ratio at the time of recombination (in units of $\left.10^{-10}\right) \eta^{C M B}=6.0_{-0.8}^{+1.1}$. This value can be used in the calculation of primordial nuclear abundances within BBN models. In the case of SBBN the knowledge of this value makes possible to predict all the primordial nuclear abundances and in particular the helium-4 $\left(Y_{p}\right)$ and the deuterium abundances that are measured in the astrophysical observations with reasonable reliability and precision. The comparison between the SBBN predictions and the measured values gives a very good agreement for 'high values' of $Y_{p}$, while there is a $4 \sigma$ discrepancy in the case of 'low values' (SBBN 'crisis'). Thus at the moment the simplest interpretation of the data is a confirmation of SBBN. However one can study how large deviations from SBBN can be if non standard BBN effects are considered. We are interested in two different classes of non standard BBN effects. The first one is the presence of extra energy density degrees of freedom that can be expressed through the extra number of neutrino species $\Delta N_{\nu}^{\rho}$. The second one is a deviation of electron neutrino and anti-neutrino distributions from the SBBN assumption of a Fermi-Dirac distribution with zero chemical potential. These two classes account for a large variety of non standard effects from theories beyond the Standard Model of fundamental interactions ${ }^{2}$. The presence of a non zero $\Delta N_{\nu}^{\rho}$ affects the standard BBN prediction on $Y_{p}$ by a quantity $\Delta Y_{p} \simeq 0.0137 \Delta N_{\nu}^{\rho}$. The effect on the Deuterium abundance is double. There is an indirect effect due to the variation of $Y_{p}$ itself, but there is also a direct effect due to change of the rate expansion induced by $\Delta N_{\nu}^{\rho}$. The distortions of electron neutrino distributions can influence only the frozen value of the neutron to proton ratio at a temperature around $0.75 \mathrm{MeV}$. When the nuclear abundances start to get synthesized at much lower temperatures around $\sim 0.065 \mathrm{MeV}$, the electron neutrinos and anti-neutrinos are fully decoupled and they do not play any role. Thus the effect of distortions of electron neutrino distributions have a direct effect only on $Y_{p}$. One can define a total effective number of extra neutrino species $\Delta N_{\nu}^{\text {tot }} \equiv\left[Y_{p}\left(\eta^{C M B}, \Delta N_{\nu}^{\rho}, \delta f_{\nu_{e}, \bar{\nu}_{e}}\right)-Y_{p}^{S B B N}\left(\eta^{C M B}\right)\right] / 0.0137$. Note that in general the distortions of the electron neutrino distribution are time dependent during the phase of neutron to proton ratio freeze-out. Note also that in general they can also give a contribution to $\Delta N_{\nu}^{\rho}$. Thus in general $\Delta N_{\nu}^{\rho}$ could change with time during BBN period. However in the models we will consider this effect is negligible and the value of $\Delta N_{\nu}^{\rho}$ can be considered constant during the BBN epoch. A comparison of the measured value $Y_{p}^{\exp }$ with $Y_{p}^{S B B N}$ can thus be translated in terms of $\Delta N_{\nu}^{\text {tot }}$ and we find, conservatively at $3 \sigma$, $-1.8<\Delta N_{\nu}^{\text {tot }}<0.3$. The possibility to distinguish separately the value of $\Delta N_{\nu}^{\rho}$ is given by a comparison between the predicted and the measured value of deuterium abundance. In this way one has a correspondence $\left(\Delta Y_{p}, \Delta(D / H)\right) \leftrightarrow\left(\Delta N_{\nu}^{\text {tot }}, \Delta N_{\nu}^{\rho}\right)$, where $\Delta$ indicates a variation compared to the SBBN case. This change of variables is not necessary in principle but makes possible to quantify in a similar way two different effects. Moreover the bi-parametrical nature of the non standard BBN effects that we are considering (we are dealing with $\eta$ as an independently measured quantity, like the neutron life time) is emphasized and this would hold considering also more than two primordial nuclear abundances (for example if one could rely primordial lithium-7 estimations).

\footnotetext{
${ }^{2}$ Another class of possible non standard effects, not considered here, is for example that one accounting for clumps or holes in baryon number density.
} 


\section{One or more sterile neutrino flavors?}

It is known since long time that the early Universe is sensitive to a mixing of the ordinary neutrinos with new possible sterile neutrinos. The simplest case is to consider a mixing between one ordinary and one sterile neutrino in isolation. In such a situation the mixing is described by just two parameters, the difference of squared masses $\delta m^{2}$ and $\sin ^{2} 2 \theta_{0}$ ( $\theta_{0}$ is the vacuum mixing angle). The task is then to calculate $\Delta N_{\nu}^{\rho}$ and $\Delta N_{\nu}^{\text {tot }}$ for any given $\left(\delta m^{2}, \sin ^{2} 2 \theta_{0}\right)$. For values $\sin ^{2} 2 \theta_{0} \gtrsim 10^{-4}$ it has been found that one always gets a positive $\Delta N_{\nu}^{\text {tot }}$. For values $\sin ^{2} 2 \theta_{0} \lesssim 10^{-4}$ and negative values of $\delta m^{2}$ the situation is quite different because a large neutrino asymmetry is generated [īin]. In the case that the ordinary neutrino is an electron neutrino and if the asymmetry is positive, then the effect on BBN is that $\Delta N_{\nu}^{\text {tot }}$ can be negative and this could be a way to reconcile standard BBN with low values of $Y_{p}$. The neutrino asymmetry generation appears as a special possibility within the simple case of two neutrino mixing. However when multiflavor mixing is considered, the much larger choice of mixing parameters makes more natural to have the right conditions of neutrino asymmetry generation being satisfied for some of the many possible sub mixings. In this case what is important is that the generated neutrino asymmetry sup-

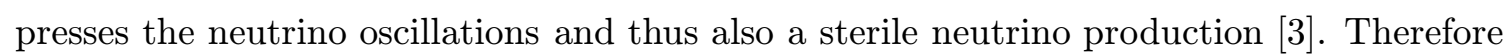
the constraints found in the two neutrino mixing can be evaded when this is embedded in a multi-flavor scenario. The neutrino asymmetry generation occurs for mixing angles much lower than those tested in the Earth experiments and so cosmology can provide a complementary information. The lower limit is given by the condition of adiabaticity. This limit

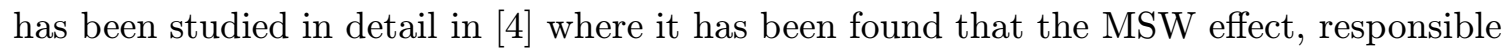
for the generation of the asymmetry, is adiabatic for $\sin ^{2} 2 \theta_{0} \gtrsim 10^{-9}\left(\mathrm{eV}^{2} /\left|\delta m^{2}\right|\right)^{1 / 4}$. The simplest realistic case of multiflavor mixing involving sterile neutrinos is a four neutrino mixing between one sterile neutrino and the three ordinary neutrinos. This is the minimal way to explain all existing experimental neutrino mixing evidences, the solar neutrino data, the atmospheric neutrino data and the short baseline LSND experiment results. This last experiment requires the existence of a $1 \mathrm{eV}^{2}$ scale for $\delta m^{2}$, much different from those that one infers for the solar and atmospheric neutrino data and thus a fourth neutrino mass and flavor eigenstate are necessary to built a neutrino mixing model consistent with all experimental data. There are two different ways to achieve this result. The first one is just by adding the sterile neutrino flavor as a sort of 'perturbation' to the three neutrino mixing matrix. In this way it almost coincides with the fourth mass eigenstate separated by the LSND gap from the other three (' $3+1$ ' models) and their mixing is such that the sterile neutrino flavor is brought to thermal equilibrium or very close to it in a way that $\Delta N_{\nu}^{\rho} \simeq 1$. The condition for a significant electron neutrino asymmetry generation able to modify the BBN predictions are not realized and therefore the final result is $\Delta N_{\nu}^{\text {tot }} \simeq 1$, incompatible with the cosmological bound. The second way represents a drastic change compared to three neutrino mixing models. In this case the four mass eigenstates are split in two couples separated by the LSND gap. The simplest realization of this class of models $(' 2+2$ ' models) is to have a full correspondence between each pair of mass eigenstates with 
a pair of neutrino flavors ${ }^{3}$. In this simplified picture, the solar neutrino data are described by oscillations of $\nu_{e}$ into $\nu_{\mu}$ or $\nu_{s}$ while the atmospheric neutrino data by oscillations of $\nu_{\mu}$ into $\nu_{\tau}$ or $\nu_{s}$. A small mixing between the two pairs has to be included to explain the LSND results but in first approximation one has four limit cases in which solar or atmospheric neutrino data (not both) are explained by oscillations of active neutrinos into sterile neutrinos. From a cosmological point of view one has to worry for those cases that include active to sterile neutrino oscillations with large mixing angles: $\nu_{\mu}$ to $\nu_{s}$ to explain atmospheric neutrino data or the LMA solution $\nu_{e}$ to $\nu_{s}$ to explain solar neutrino data. One can again slightly perturb these 4 limit cases in order to get a small mixing between the two couples such to generate a neutrino asymmetry that suppresses the sterile neutrino production [int. However the most recent analysis for the atmospheric and solar neutrino data taken separately, disfavor approximately at $3 \sigma$ the possibility of pure active to sterile neutrino oscillations. In a joint four neutrino mixing analysis of solar and atmospheric neutrino data, the possibility to have pure active to sterile neutrino oscillations in one of the two cases is only slightly disfavored but the best fit is obtained for those models in which the muon neutrinos are converted into a mixture of sterile and tau neutrinos in the atmospheric case and the electron neutrinos into a mixture of ordinary (muon and tauon) and sterile neutrinos in the solar case [6]. In this situation the sterile neutrino flavor is brought to thermal equilibrium and moreover a neutrino asymmetry generation able to suppress the sterile neutrino production or to yield a negative contribution to $\Delta N_{\nu}^{\text {tot }}$ is not possible. Therefore, like for $3+1$ models, one gets again $\Delta N_{\nu}^{\text {tot }} \simeq 1$, at variance with the cosmological bound. Future data should be able to constraint even more the possibility of pure active to sterile neutrino oscillations and thus the possibility to have a neutrino asymmetry generation in four neutrino mixing models. If this will be the case then the possibility to include the LSND results in a consistent four neutrino mixing picture not conflicting with the cosmological observations relies on the assumption that large neutrino asymmetries were generated at temperatures much above $10 \mathrm{MeV}$ ('pre-existing' neutrino asymmetries). This would be an important piece of information for models of baryo-leptogenesis. Another possibility is to assume the existence of a second sterile neutrino flavor mixed with the ordinary ones with the appropriate mixing parameters such that a positive electron neutrino asymmetry is generated. This would both result in a negative contribution to $\Delta N_{\nu}^{\text {tot }}$ and at the same time would halve the sterile neutrino production in a way to have $\Delta N_{\nu}^{\rho} \simeq 0.5$ and $\Delta N_{\nu}^{\text {tot }}<0.3$. In this way it would be possible to reconcile the LSND result with cosmology. For this to happen the electron neutrino flavor should be mainly 'contained' in the $1 \mathrm{eV}$ mass eigenstates. If this is true the next generation of $\beta \beta_{0 \nu}$ experiments could be able, under some conditions, to detect a neutrino mass. Neutrino mixing models with more than one sterile neutrino flavors arise, for example, in theories with large extra dimensions. In this case the role of sterile neutrinos is played by the Kaluza Klein states of one fermion living on the bulk and mixed with the ordinary neutrino on the brane. Unfortunately all models proposed up to now do not seem to solve the problems of four neutrino mixing models.

\footnotetext{
${ }^{3}$ It corresponds to have a $2 \times 2$ block factorization of the mixing matrix.
} 


\section{Possible future scenarios}

In future years both cosmological observations and neutrino mixing experiments will provide many new data. From the CMB power spectrum the Planck satellite should be able to measure the baryon content with a $1 \%$ error and at the same to detect $\Delta N_{\nu}^{\rho}$ with a realistic error of 0.1. All these new data will be able to constraint very strongly the possibility of non standard BBN effects from active-sterile neutrino mixing. We can list the possible scenarios, assuming that pure active to sterile neutrino oscillations will be definitively ruled out both in solar and atmospheric neutrino data, that means to exclude the case that four neutrino mixing models are compatible with cosmology without assuming pre-existing large neutrino asymmetries. 1) The LSND result is confirmed but non SBBN effects are not found, even though expected. A likely explanation would be the assumption of pre-existing large neutrino asymmetries. 2) LSND is confirmed but non SBBN effects are discovered and this means $\left(\Delta N_{\nu}^{\rho}, \Delta N_{\nu}^{\text {tot }}\right) \neq(0,0)$. A result $\Delta N_{\nu}^{\text {tot }}<\Delta N_{\nu}^{\rho}$ would be a specific signature of electron neutrino asymmetry generation and thus of active-sterile neutrino mixing. In this case one can conclude that there are additional sterile neutrino flavors other than that one suggested by the LSND experiment. If one can attribute the effects to neutrino mixing, then one can also remarkably conclude that pre-existing neutrino asymmetries were small. 3) Non SBBN effects are found but LSND is disproved. In this case the problem to attribute the effects to active-sterile neutrino mixing would be crucial. As we said a result $\Delta N_{\nu}^{\text {tot }}<\Delta N_{\nu}^{\rho}$ would represent a good signature. The possible formation of neutrino domains associated to the neutrino asymmetry generation $[\bar{i}]$ would have effects [is] that could provide other kind of signatures. 4) LSND is disproved and cosmological observations do not find any non SBBN effect. In this case a three ordinary neutrino mixing will be enough to explain all the data and it will be challenging to search for cosmological effects of it.

\section{Acknowledgments}

P. Di Bari is an Alexander von Humboldt Foundation fellow. He thanks M. Lusignoli and L. Mersini for valuable discussions.

\section{References}

[1] P. Di Bari, hep-ph/0108182, to appear on Phys. Rev. D.

[2] R. Foot, M. J. Thomson and R. R. Volkas, Phys. Rev. D 53 (1996) 5349.

[3] R. Foot and R. R. Volkas, Phys. Rev. Lett. 75 (1995) 4350; P. Di Bari, P. Lipari and M. Lusignoli, Int. J. Mod. Phys. A 15 (2000) 2289.

[4] P. Di Bari and R. Foot, arXiv:hep-ph/0103192, to appear on Phys. Rev. D.

[5] N. F. Bell, R. Foot and R. R. Volkas, Phys. Rev. D 58 (1998) 105010.

[6] M. C. Gonzalez-Garcia, M. Maltoni and C. Pena-Garay, these proceedings, hep-ph/0108073.

[7] P. Di Bari, Phys. Lett. B 482 (2000) 150.

[8] A. D. Dolgov and D. Grasso, arXiv:astro-ph/0106154. 